\title{
Les traumatismes crâniens font partie des blessures associées au curling récréatif qui peuvent être évitées en portant des chaussures appropriées
}

\author{
D. K. Ting, M.D. (1); R. J. Brison, M.D., M.H.P. (2)
}

Cet article a fait l'objet d'une évaluation par les pairs.

Diffuser cet article sur Twitter

\section{Résumé}

Introduction : Notre étude s'intéresse à une population de joueurs de curling et vise à décrire la fréquence des blessures, à estimer le risque de se blesser et à évaluer l'opinion des joueurs à l'égard des stratégies de prévention axées sur l'équipement.

Méthodologie : Dans le cadre d'une étude de série de cas rétrospective, nous avons fait des recherches dans le Système canadien hospitalier d'information et de recherche en prévention des traumatismes (SCHIRPT), une base de données nationale pour la surveillance des blessures, en vue d'y repérer les blessures subies au curling répertoriées entre 1993 et 2011. L’Hôpital général de Kingston et l'Hôpital Hôtel-Dieu sont les deux hôpitaux de Kingston (Ontario) qui offrent des services d'urgence et qui font partie du réseau du SCHIRPT. Pour chaque entrée trouvée, nous avons procédé à l'analyse du dossier du patient. Un sondage complémentaire a été envoyé à certaines personnes ayant subi des blessures au curling afin d'en connaitre les détails et de savoir ce qu'elles pensent du port d'un équipement comme mesure de prévention. Nous avons utilisé des statistiques descriptives pour les taux et les proportions.

Résultats : Plus de $90 \%$ des blessures graves au curling résultent d'une chute et 31,7 \% des cas de blessures graves sont constitués de choc à la tête. Nous avons calculé que le taux de blessures graves qui nécessitent une consultation aux services d'urgence est approximativement de 0,17 pour 1000 athlètes exposés (intervalle de confiance à $95 \%$ : de 0,12 à 0,22 ). Le sondage complémentaire a été rempli par $54 \%$ des joueurs blessés ciblés. Parmi eux, 41,3\% attribuent leur chute à l'absence de chaussures appropriées. Concernant les stratégies de prévention, 73,5 $\%$ des répondants étaient d'accord avec la suggestion du port obligatoire de chaussures spécialisées, mais seulement $8 \%$ avec celle du port obligatoire du casque.

Conclusion : Bien que les blessures subies au curling qui nécessitent des soins médicaux ne soient pas courantes, les traumatismes crâniens représentent une proportion importante de ces blessures. Le port obligatoire de chaussures appropriées semble constituer la mesure de prévention la plus efficace et la plus acceptable pour les joueurs.

Mots-clés : curling, blessure résultant de la pratique des sports, commotion cérébrale, prévention des blessures, médecine d'urgence

\section{Introduction}

Le curling est un sport de compétition et de loisir populaire, qui fait partie intégrante de l'identité canadienne ${ }^{1-3}$. Le curling est devenu un sport olympique officiel lors des Jeux olympiques d'hiver de 1998. Bien que la Fédération mondiale de curling

\section{Principales constatations}

- Bien que le curling soit un sport populaire au Canada, nous ne savons pas vraiment dans quelle mesure les blessures sont fréquentes chez ceux qui s'adonnent à ce sport à des fins récréatives.

- Nous examinons dans cette étude la nature anatomique des blessures subies et la façon dont ces blessures se sont produites chez les joueurs de curling récréatif qui se sont présentés pour obtenir des soins dans un service d'urgence qui fait partie du réseau du SCHIRPT à Kingston (Ontario).

- Plus de $90 \%$ des blessures étaient liées à des chutes non contrôlées sur la glace, et les traumatismes crâniens (dont des commotions) ainsi que les contusions et les fractures des membres supérieurs représentaient les principales lésions anatomiques observées.

- Un sondage parmi les joueurs blessés a révélé que ceux-ci sont largement favorables au port de chaussures appropriées comme stratégie de prévention clé, mais non au port du casque.

compte 49 pays membres, les Canadiens représentent environ $80 \%$ de la population de joueurs de curling dans le monde, avec une population de joueurs variant entre 730000 et 870000 adeptes $^{1,2,4}$. Malgré cela, il y a très peu de données publiées concernant la fréquence des blessures.

Rattachement des auteurs :

1. Département de médecine d'urgence, Université de la Colombie-Britannique, Kelowna (Colombie-Britannique), Canada

2. Centre de recherche clinique de l'Hôpital général de Kingston, Département de médecine d'urgence, Université Queen's, Kingston (Ontario), Canada

Correspondance : Daniel Ting, Hôpital général de Kelowna, 2312, rue Pandosy, Kelowna (Colombie-Britannique) V1Y 1T3; tél. : 250-980-1343; téléc. : 250-980-1356;

courriel : daniel.ting@alumni.ubc.ca 
Le curling se joue sur la glace. Le but du jeu est de faire glisser une pierre de 20 kilogrammes sur une surface glacée et de l'amener à s'immobiliser le plus près possible d'une cible. Deux équipes, chacune composée de quatre joueurs, s'efforcent, à tour de rôle, de lancer stratégiquement les pierres. Les membres de chaque équipe modifient le parcours de la pierre à l'aide de balais de curling, qu'ils utilisent pour balayer la piste afin de faire fondre la glace autour de la pierre, ce qui réduit le frottement.

Les risques de blessures au curling sont nombreux. Étant donnée que le caractère glissant de la glace est mis à profit dans le jeu, l'équilibre, la souplesse et une certaine expérience sont nécessaires pour réduire le risque de chute. Le balayage doit être vigoureux et nécessite de la part des joueurs de la coordination pour glisser et éviter les obstacles $^{5}$. La maîtrise de ces techniques et de ces compétences peut nécessiter des années de pratique intensive.

Le curling est généralement perçu comme un sport présentant peu de risques de se blesser, quoique les données publiées soient limitées et portent plutôt sur la population des joueurs de compétition. Une étude axée sur les deux championnats nationaux a révélé que le taux autodéclaré de blessures graves entraînant une " perte de temps », ce qui caractérise l'exposition de l'athlète, était de 2 à 2,3 par 1000 parties jouées ${ }^{4}$. Lors d'un autre championnat masculin, le taux autodéclaré de blessures graves a été de 3,5 par 1000 athlètes exposés ${ }^{6}$. Lors des Jeux olympiques d'hiver de 2010, le taux de blessures ayant nécessité des soins médicaux était de 2,5 par 1000 athlètes exposés, les 2 cas recensés étant survenus chez les hommes et aucun incident du même genre n'ayant été signalé chez les femmes ${ }^{7}$. La plupart des blessures rapportées étaient des entorses au genou, au dos et à l'épaule, et aucun traumatisme crânien n'a été expressément déclaré.

Un équipement spécialisé permet de maximiser la performance et de réduire le risque de blessures chez les joueurs de curling. Il est constitué d'un balai, que les joueurs utilisent pour balayer la piste et pour garder leur équilibre au moment de " placer la pierre », et des souliers de curling spécialisés, à la fois « antidérapants » et " glisseurs ». Ces souliers, en plus d'améliorer la performance des joueurs de curling, réduisent leur risque de glissade lorsqu'ils se déplacent sur la piste. Alors que les balais sont utilisés par tous, il arrive souvent que les clubs de curling n'obligent pas ceux qui jouent à des fins récréatives à porter des chaussures appropriées. Ainsi, certains de ces joueurs portent des chaussures de course plutôt que des souliers antidérapants et, plutôt que d'utiliser un glisseur approprié, ils recouvrent les semelles de leurs souliers avec du ruban pour pouvoir glisser. On ne porte habituellement pas de casque protecteur au curling.

Plusieurs questions importantes concernant les blessures subies au curling restent toujours sans réponse. Toutes les études réalisées jusqu'à maintenant ont mis l'accent sur les joueurs de compétition et d'élite, qui constituent une faible proportion des curleurs et qui ont vraisemblablement un profil de blessures différent de celui des joueurs de curling récréatif. De plus, ces études ont porté sur des effectifs de curleurs assez réduits (100 joueurs ou moins) et n'ont permis de recueillir des renseignements qu'à une date précise. Enfin, nous ne savons rien de l'opinion des curleurs à propos des stratégies de prévention visant l'équipement, alors que cette opinion est essentielle pour prévenir efficacement les blessures liées à la pratique sportive ${ }^{8}$.

Notre étude de prévalence et d'observation a une triple portée : décrire le type et la gravité des blessures subies par les joueurs de curling récréatif, estimer le taux de blessures graves subies par une population de joueurs de curling récréatif pendant la pratique de leur sport et sur une période de plusieurs années et évaluer l'attitude des curleurs à l'égard du port de l'équipement visant la prévention des blessures.

Cette recherche a été approuvée d'un point de vue éthique par le Comité d'éthique de la recherche en sciences de la santé de l'Université Queen's à Kingston (Ontario, Canada).

\section{Méthodologie}

\section{Lieu de l'étude}

Kingston compte trois clubs de curling : les clubs Royal Kingston, Cataraqui et Garrison.
La collectivité de Kingston compte deux hôpitaux : l'Hôpital général de Kingston et l'Hôpital Hôtel-Dieu. Ce sont les seuls hôpitaux offrant des services d'urgence à Kingston, et ces deux établissements accueillent à la fois adultes et enfants. Un service de radiodiagnostic est offert dans les deux hôpitaux et, contrairement à bon nombre d'autres villes canadiennes, il n'y avait dans cette ville aucune clinique sans rendez-vous offrant des services de radiologie durant la période à l'étude.

\section{Population à risque}

La population à risque a été estimée à partir du nombre de joueurs membres des 3 clubs de curling de Kingston, qui comptaient au total 1184 membres en 2012 (tableau 1). Nous avons supposé que le nombre local de membres est demeuré stable pendant la période visée par l'étude. Les données ont été recueillies en communiquant personnellement avec les administrateurs responsables des adhésions. Sport Canada rapporte des taux nationaux de participation au curling relativement stables, soit $1,3 \%$ en $1998^{9}$, $1,1 \%$ en $2005^{10}$ et $0,9 \%$ en $2010^{11}$. Nous avons également présumé que les curleurs blessés qui se sont présentés à l'Hôpital général de Kingston ou à l'Hôpital HôtelDieu étaient membres de l'un des clubs de curling de Kingston. La répartition par groupe d'âge a été la suivante : 17 ans et moins, de 18 à 34 ans, de 35 à 49 ans, de 50 à 64 ans et 65 ans et plus.

\section{Base de données du Système canadien hospitalier d'information et de recherche en prévention des traumatismes et analyse du dossier des patients}

Le Système canadien hospitalier d'information et de recherche en prévention des

TABLEAU 1

Nombre de joueurs membres des clubs de curling de Kingston en 2012, selon l'âge et le sexe des joueurs

\begin{tabular}{lccr} 
Âge & Hommes & Femmes & Total \\
\hline 17 ans et moins & 83 & 36 & 119 \\
De 18 à 34 ans & 53 & 27 & 80 \\
De 35 à 49 ans & 70 & 65 & 135 \\
De 50 à 64 ans & 259 & 246 & 505 \\
65 ans et plus & 218 & 127 & 345 \\
Total & 683 & 501 & 1184 \\
\hline
\end{tabular}


traumatismes (SCHIRPT) est une base de données créée en 1990 pour assurer la surveillance des blessures et fondée sur les soins offerts dans les services d'urgence ${ }^{12}$. Quinze hôpitaux y participent au Canada (11 hôpitaux pédiatriques et 4 hôpitaux généraux). Lorsque des patients blessés se présentent aux services d'urgence d'un hôpital participant, ils sont invités à remplir un questionnaire d'une page dans lequel ils doivent décrire les circonstances entourant l'incident et la façon dont leurs blessures se sont produites, et ils doivent fournir aussi des données individuelles. Le personnel de recherche consigne les renseignements concernant la nature des blessures subies et des traitements administrés. L'Hôpital général de Kingston et l'Hôpital Hôtel-Dieu font tous deux partie du réseau du SCHIRPT.

Nous avons utilisé deux stratégies pour cibler les participants potentiels à notre étude. D'une part, un code particulier dans la base de données du SCHIRPT indique que le curling est l'activité à l'origine de la blessure. D'autre part, nous avons effectué une recherche textuelle en vue de trouver les lettres " curl » dans la description de la blessure. Nous avons recherché les blessures survenues au cours d'une période de 18 ans, du $1^{\text {er }}$ octobre 1993 au 28 février 2011. Nous avons ensuite examiné chaque entrée trouvée et avons supprimé celles qui se répétaient ou qui n'étaient pas pertinentes. Nous avons tenté de cibler les cas de blessures graves au curling et avons omis ceux où la pratique de ce sport avait entraîné des problèmes de santé chroniques.

Après avoir ciblé les participants potentiels à l'étude, nous avons examiné le dossier médical de ces patients afin d'obtenir des renseignements supplémentaires concernant la façon dont l'accident est survenu, la partie du corps touchée, la gravité de la blessure et le traitement administré.

\section{Sondagé complémentaire}

Nous avons repéré les curleurs ayant subi une blessure au cours de la dernière décennie pour laquelle des données étaient disponibles (période se terminant le 28 février 2011). Nous avons examiné leurs dossiers médicaux afin d'identifier les curleurs dont le décès avait été confirmé pour éviter de les inclure dans notre sondage. Nous avons obtenu ainsi 104 curleurs admissibles, à qui nous avons envoyé par la poste un questionnaire accompagné d'une description des objectifs du sondage. Dans ce sondage, les participants étaient invités à fournir la cause de la blessure et à dire comment elle s'était produite, à exprimer leur opinion concernant les stratégies de prévention des blessures axées sur l'équipement, à nommer d'autres blessures subies au curling et à fournir quelques caractéristiques individuelles. Un rappel a été envoyé 6 semaines plus tard à ceux qui n'avaient toujours pas répondu au sondage. Aucune mesure incitative n'était offerte pour encourager les réponses.

\section{Analyse statistique}

Le taux de blessures a été calculé en divisant le nombre de blessures graves par le taux d'exposition des athlètes de la population à risque (tableau 2). L'exposition des athlètes a été calculée à partir des données du sondage complémentaire : le nombre de parties jouées par semaine a été multiplié par la durée de la saison de curling. La saison de curling a été estimée comme étant les mois au cours desquels nous avons observé plus de 10 blessures. La signification statistique a été évaluée au moyen de tests du chi carré. Pour que l'hypothèse nulle soit rejetée, la probabilité devait être de $p<0,05$.

\section{Résultats}

\section{Taux de blessures graves}

Nous avons ciblé 223 personnes ayant subi des blessures au curling. De ce nombre, 208 ont été considérées comme ayant souffert de blessures graves et non de lésions chroniques (tableau 3). Six

\section{TABLEAU 2}

Expérience de la pratique du curling des répondants au sondage complémentaire au moment où ils ont été blessés

\begin{tabular}{lrlll}
\multicolumn{2}{l}{$\begin{array}{c}\text { Exposition des athlètes } \\
\text { par semaine }(\%)\end{array}(\mathbf{n}=49)$} & \multicolumn{2}{c}{$\begin{array}{c}\text { Années d'expérience } \\
\text { au curling }(\%)\end{array}(\mathbf{n}=48)$} \\
\hline Moins de 1 fois & 22 & Moins de 1 an & 17 \\
De 1 à 2 fois & 39 & De 1 à 3 ans & 17 \\
De 3 à 4 fois & 35 & De 4 à 6 ans & 13 \\
Plus de 4 fois & 4 & 7 ans ou plus & 54 \\
\hline
\end{tabular}

TABLEAU 3

Caractéristiques des curleurs qui se sont présentés aux services d'urgence pour une blessure grave, 1993 à 2011

\begin{tabular}{lccc} 
Âge & Hommes & Femmes & Total \\
\hline 17 ans et moins & 6 & 7 & 13 \\
De 18 à 34 ans & 15 & 23 & 38 \\
De 35 à 49 ans & 19 & 23 & 42 \\
De 50 à 64 ans & 21 & 36 & 57 \\
65 ans et plus & 37 & 21 & 58 \\
Total & 98 & 110 & 208 \\
\hline
\end{tabular}

athlètes avaient subi 2 blessures différentes. Le plus jeune curleur blessé était âgé de 7 ans. Nous avons observé des blessures importantes au curling pendant 7 mois de l'année. D’après le sondage complémentaire, le taux moyen d'exposition des athlètes était de 2,1 par semaine (tableau 2). Nous estimons que le taux de blessures graves ayant nécessité une consultation à l'urgence est de 0,17 par 1000 athlètes exposés (intervalle de confiance à $95 \%$ : de 0,12 à 0,22 ).

\section{Caractérisation des blessures}

Le tableau 4 détaille les blessures. Parmi les blessures graves observées, 92,7\% étaient attribuables à une chute sur la glace. Deux comportements types ont été observés en ce qui concerne les chutes : glisser et tomber à la renverse, ce qui occasionne un traumatisme crânien dans la région occipitale $(28,1 \%)$, ou glisser et tomber vers l'avant, ce qui entraine des blessures causées par une chute sur un bras en extension (20,8\%). C'est ainsi que se produisent $49,0 \%$ des chutes.

Conformément à ces mécanismes, les traumatismes crâniens formaient la catégorie de blessures la plus courante à la suite d'une chute, suivis des blessures aux poignets ou aux mains : ensemble, ces blessures comptaient pour 54,3\% du total des blessures graves (figure 1). Les blessures causées par un choc à la tête comprenaient 37 commotions ou traumatismes crâniens fermés (tableau 4), et 19,3\% des blessures résultant d'une chute.

\section{Attitude à l'égard des stratégies de prévention des blessures}

Sur les 104 sondages envoyés par la poste, 13 nous ont été retournés sans même 
TABLEAU 4

Liste des blessures graves au curling par région anatomique

\begin{tabular}{|c|c|c|c|}
\hline $\begin{array}{l}\text { Partie du corps } \\
\text { touchée }\end{array}$ & Description & $\begin{array}{c}\text { Nombre de blessures } \\
\text { recensé }\end{array}$ & $\%$ \\
\hline \multicolumn{4}{|l|}{ Tête } \\
\hline \multirow[t]{3}{*}{ Cerveau } & Traumatisme crânien fermé ou commotion & 37 & 17,8 \\
\hline & Lésion nerveuse & 1 & 0,5 \\
\hline & Hémorragie intracrânienne & 3 & 1,4 \\
\hline Crâne & Lésion des tissus mous & 12 & 5,8 \\
\hline Visage & Lésion des tissus mous & 13 & 6,3 \\
\hline \multicolumn{4}{|l|}{ Tronc } \\
\hline \multirow[t]{2}{*}{ Thorax } & Lésion des tissus mous & 9 & 4,3 \\
\hline & Fracture & 8 & 3,8 \\
\hline Bas du dos & Lésion des tissus mous & 5 & 2,4 \\
\hline \multicolumn{4}{|l|}{ Haut du bras } \\
\hline \multirow[t]{2}{*}{ Épaule } & Lésion des tissus mous & 13 & 6,3 \\
\hline & Luxation/fracture & 9 & 4,3 \\
\hline \multirow[t]{2}{*}{ Coude } & Lésion des tissus mous & 10 & 4,8 \\
\hline & Fracture & 5 & 2,4 \\
\hline \multicolumn{4}{|l|}{ Poignet ou main } \\
\hline \multirow[t]{2}{*}{ Poignet } & Lésion des tissus mous & 11 & 5,3 \\
\hline & Fracture & 23 & 11,1 \\
\hline \multirow[t]{2}{*}{ Main } & Lésion des tissus mous & 8 & 3,8 \\
\hline & Luxation/fracture & 5 & 2,4 \\
\hline \multicolumn{4}{|l|}{ Membres inférieurs } \\
\hline \multirow[t]{2}{*}{ Hanche } & Lésion des tissus mous & 3 & 1,5 \\
\hline & Fracture & 5 & 2,4 \\
\hline \multirow[t]{2}{*}{ Genou } & Lésion des tissus mous & 9 & 4,3 \\
\hline & Luxation ou fracture & 5 & 2,4 \\
\hline Jambe & Fracture & 3 & 1,4 \\
\hline \multirow[t]{2}{*}{ Cheville ou pied } & Lésion des tissus mous & 7 & 3,4 \\
\hline & Fracture & 4 & 1,9 \\
\hline Total & & 208 & 100,0 \\
\hline
\end{tabular}

avoir été ouverts. Sur les 91 personnes qui, selon nous, ont reçu le sondage, 49 l'ont retourné après l'avoir rempli (soit $54 \%)$. Le profil de la population de répondants n'était pas très différent de celui de la population globale en ce qui a trait aux groupes d'âge ou au sexe $(p>0,05)$.

Bon nombre des joueurs blessés étaient des curleurs expérimentés : la majorité d'entre eux (54\%) possédaient au moins 7 années d'expérience dans la pratique du curling et y jouaient de 1 à 4 fois par semaine pendant la saison (74 \%) (tableau 3).

En conformité avec les résultats tirés de l'ensemble complet de données, les répondants ont attribué plus de $90 \%$ de leurs blessures à des chutes sur la glace, le plus souvent du fait de l'absence de chaussures de curling appropriées. En fait, 41,3 \% des répondants ont attribué leur chute à l'absence de chaussures de curling appropriées. L'idée de rendre obligatoire le port de chaussures appropriées a été approuvée par près des trois quarts des répondants (tableau 5).

L'une des questions du sondage portait sur l'avis des participants à propos du port du casque durant le jeu de curling. Quelques répondants (8\%) étaient d'avis que le port du casque devrait être obligatoire, mais $85 \%$ d'entre eux étaient d'avis que le casque devrait être porté dans certaines circonstances seulement (p. ex. par les
FIGURE 1

Parties du corps touchées lors de blessures graves au curling, réparties par région anatomique

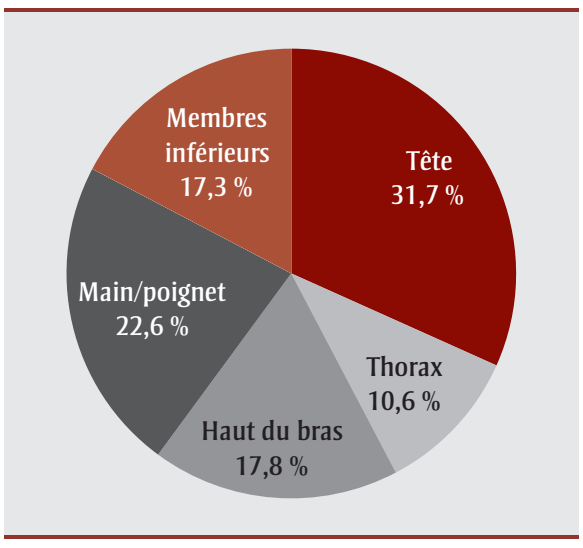

débutants, les jeunes enfants, les curleurs ayant une limitation fonctionnelle) ou simplement recommandé de façon générale (tableau 5).

Les curleurs interrogés n'étaient pas vraiment favorables à l'utilisation de protègepoignets similaires à ceux utilisés par les adeptes de la planche à neige.

Bien que la consommation d'alcool soit associée accessoirement au curling, nous n'avons pas constaté qu'elle ait augmenté significativement le risque de blessures graves. Seul l'un des répondants était d'avis que l'alcool avait contribué à une blessure. Dans le même ordre d'idées, seulement trois des dossiers médicaux et de la base de données du SCHIRPT mentionnaient l'alcool comme l'un des facteurs.

TABLEAU 5

Attitude des répondants au sondage complémentaire à l'égard des stratégies de prévention des blessures au curling

\begin{tabular}{|c|c|c|}
\hline & $\begin{array}{l}\text { Port du } \\
\text { casque }(\%) \\
(\mathrm{n}=48)\end{array}$ & $\begin{array}{l}\text { Port de } \\
\text { chaussures de } \\
\text { curling (\%) } \\
(\mathrm{n}=49)\end{array}$ \\
\hline Aucune utilité & 6 & 0 \\
\hline $\begin{array}{l}\text { À porter dans certaines } \\
\text { circonstances }\end{array}$ & 50 & 8 \\
\hline $\begin{array}{l}\text { Usage général } \\
\text { recommandé }\end{array}$ & 35 & 18 \\
\hline Port obligatoire & 8 & 74 \\
\hline
\end{tabular}




\section{Analyse}

Notre étude rétrospective d'une série de cas a révélé que les blessures subies par la population de joueurs de curling récréatif étaient principalement attribuables à une chute, soit car le joueur était tombé vers l'avant et s'était blessé au poignet, soit qu'il était tombé à la renverse et avait subi un traumatisme crânien. Nous avons également constaté que les blessures chez les curleurs peuvent être graves et qu'elles peuvent entraîner des lésions cérébrales, ce qui est plus troublant. Le taux de blessures graves nécessitant une consultation aux services d'urgence au sein de la population de joueurs de curling récréatif était de 0,17 par 1000 athlètes exposés. Le port de chaussures de curling appropriées n'est pas fermement ancré dans la culture des joueurs de curling récréatif : les curleurs ont indiqué que l'absence de ce type de chaussures est l'une des principales causes de chutes.

Environ le tiers des chutes ont causé un traumatisme crânien. Les ouvrages sur le curling de compétition ne font pas expressément mention des traumatismes crâniens, ce qui donne à penser que ce risque est plus élevé au sein de la population des joueurs de curling récréatif. Cela est logique, étant donné que ces derniers, en particulier les débutants, sont moins à l'aise pour glisser sur la glace et sont plus susceptibles de perdre l'équilibre et de tomber. De la même manière, les chutes vers l'arrière qui causent un choc à la tête ont été observées plus souvent chez les surfeurs des neiges débutants que chez leurs homologues expérimentés ${ }^{13}$.

Récemment, la communauté scientifique a accordé une plus grande attention aux séquelles laissées par les commotions résultant de la pratique d'un sport ${ }^{14-15}$. Toutefois, l'efficacité des différentes méthodes utilisées pour se protéger la tête reste encore incertaine. Bien que les casques réduisent le risque de subir un traumatisme crânien moyen ou grave, il peut ne pas empêcher les commotions, compte tenu de la façon dont ils sont conçus actuellement ${ }^{16-18}$. En outre, bien que notre sondage ait révélé que la plupart des curleurs considèrent les casques comme importants, ceux qui le portent à l'heure actuelle sont rares et stigmatisés. Devant ce type de problème, d'autres sports ont modifié leurs règlements afin de réduire les commotions ${ }^{13}$. Au curling, on pourrait adopter une mesure visant à rendre obligatoire le port de chaussures de curling, qui offrent un meilleur contrôle sur la glace et qui peuvent donc prévenir les chutes et, de ce fait, les traumatismes crâniens qui en résultent. Comme près de la moitié des répondants à notre sondage ont attribué leur chute à l'absence de chaussures appropriées, les clubs de curling pourraient facilement rendre obligatoire le port de chaussures adéquates ou du moins en mettre à la disposition des joueurs sur place.

À Kingston, l'un des clubs de curling a instauré une politique qui oblige les curleurs de 11 ans et moins à porter un casque. Dans notre ensemble de données, 8 blessés étaient âgés de moins de 11 ans. Les ouvrages consultés donnent à penser que les jeunes athlètes qui subissent une commotion prennent plus de temps à se rétablir et n'ont pas les mêmes réactions physiologiques que les athlètes plus âgés ${ }^{19-21}$. Selon d'autres recherches, les femmes semblent aussi plus susceptibles de subir une commotion et nécessiter une période de rétablissement plus longue ${ }^{22-24}$. Par conséquent, il serait approprié d'adopter des stratégies de prévention plus agressives auprès de ces groupes, de même qu'auprès des adultes débutants.

\section{Points forts et limites}

Bien que notre étude n'ait mis en lumière qu'un nombre relativement peu élevé de blessures subies au curling et n'ait permis qu'une analyse descriptive, il s'agit de l'examen le plus complet réalisé jusqu'à maintenant sur les blessures graves au curling et de la première étude à décrire les blessures subies par les joueurs de curling récréatif. Nous avons eu recours à un programme de surveillance des blessures déjà bien établi relativement aux soins prodigués dans les services d'urgence, qui permet de cibler toutes les personnes blessées qui se présentent dans l'un des deux établissements qui offrent leurs services à des populations distinctes sur le plan géographique. Compte tenu de la fréquence peu élevée des blessures, il aurait été difficile de réaliser une étude prospective. Nous avons également effectué un sondage complémentaire afin de compléter les données disponibles dans le SCHIRPT et de connaître l'opinion des joueurs à l'égard des stratégies de prévention des blessures. Nous avons établi que les traumatismes crâniens représentent une portion importante des blessures graves au curling et, en fonction des réponses au sondage, nous avons déterminé une stratégie de prévention simple : le port obligatoire de chaussures de curling.

Toutefois, nos résultats découlent d'une étude rétrospective de série de cas et d'une approximation de la population à risque. Il est probable que nous ayons fait des estimations prudentes en ce qui a trait aux blessures, étant donné que nous avons recherché uniquement les personnes blessées qui ont obtenu des soins dans des services d'urgence.

Notre sondage complémentaire a été rempli par seulement $54 \%$ de ceux à qui nous l'avons envoyé. Cet échantillon de petite taille a été utilisé pour estimer l'exposition au risque chez les joueurs de curling récréatif. En outre, bien que le profil des répondants au sondage corresponde à celui de l'ensemble de la population de curleurs de Kingston pour ce qui est du sexe et de l'âge des joueurs, tous les curleurs interrogés ont été blessés, ce qui signifie qu'ils sont peut-être plus enclins à approuver les mesures de sécurité que ceux qui n'ont subi aucune blessure. Tous les répondants au sondage ont en tout cas convenu que les joueurs devraient porter des chaussures mieux adaptées lorsqu'ils pratiquent le curling, et presque les trois quarts d'entre eux ont approuvé le port obligatoire de chaussures appropriées, ce qui donne à penser que la population de curleurs en général réagirait favorablement à cette décision.

\section{Conclusion}

Notre étude, fondée sur un programme de surveillance des blessures bien établi (le SCHIRPT), est la première à présenter des données sur la fréquence et la typologie des blessures chez les joueurs de curling récréatif. La plupart des blessures surviennent à la suite d'une chute, et l'une des 
principales causes signalées est l'absence de chaussures appropriées de curling.

Nous recommandons de rendre obligatoire le port de chaussures de curling appropriées et que les clubs de curling revoient leurs politiques en ce qui concerne la protection de la tête, en particulier chez les débutants et les jeunes curleurs. Les consultations liées à la pratique du curling peuvent être une bonne occasion pour les médecins cliniciens qui travaillent aux services d'urgence d'encourager les joueurs à porter des chaussures appropriées pour éviter d'autres blessures.

D'autres études pourraient traiter également du lien entre expertise des curleurs et blessures subies afin de mieux définir les cas où il serait le plus profitable de porter des chaussures de curling et un casque. Un sondage pourrait être mené auprès de tous les curleurs pour connaître leur position vis-à-vis des stratégies axées sur l'équipement et pour mieux caractériser ainsi l'opinion de ces athlètes.

\section{Remerciements}

Cette étude a été rendue possible grâce au soutien de l'Agence de la santé publique du Canada. Les résultats préliminaires de cette recherche ont été présentés lors de la conférence de 2013 de l'Association canadienne des médecins d'urgence tenue à Vancouver (Colombie-Britannique, Canada).

\section{Références}

1. Leipert BD, Plunkett R, Meagher-Stewart D, Scruby L, Mair H, Wamsley KB. «Je ne peux pas imaginer ma vie sans ça! » Le curling et la promotion de la santé : une étude réalisée à l'aide de la méthode photovoix. Revue canadienne de recherche en sciences infirmières. 2011;43(1):60-78.

2. Mair H. Curling in Canada: from gathering place to international spectacle. Int J Can Stud. 2007;35:39-60.

3. Wieting SG, Lamoreux D. Curling in Canada. Cult Sport Soc. 2001;4(2):140-156.
4. Reeser JC, Berg RL. Self reported injury patterns among competitive curlers in the United States: a preliminary investigation into the epidemiology of curling injuries. Br J Sports Med. 2004;38(5):E29.

5. Bradley JL. The sports science of curling: a practical review. J Sport Sci Med. 2009 Dec 1;8(4):495-500.

6. Berry JW, Romanick MA, Koerber SM. Injury type and incidence among elite level curlers during world championship competition. Res Sports Med. 2013;21(2):159-163. doi: 10.1080/15438627.2012.757229

7. Engebretsen L, Steffen K, Alonso JM et collab. Sports injuries and illnesses during the Winter Olympic Games 2010. Br J Sports Med. 2010;44(11):772-780. doi: 10.1136/bjsm.2010 .076992

8. Finch CF, Donaldson A. A sports setting matrix for understanding the implementation context for community sport. Br J Sports Med. 2010;44(13):973-978. doi: 10.1136/bjsm .2008.056069

9. Sport Canada. La pratique des sports au Canada, Rapport de 1998. Ottawa (Ont.) : Culture, Tourisme et Centre de la statistique de l'éducation, Statistique Canada; 2000.

10. Ifedi F. La pratique des sports au Canada, 2005. Ottawa (Ont.) : Culture, Tourisme et Centre de la statistique de l'éducation, Statistique Canada; 2008.

11. Patrimoine canadien. Participation au sport 2010. Ottawa (Ont.): Patrimoine canadien; 2013.

12. Mackenzie SG, Pless IB. CHIRPP: Canada's principal injury surveillance program. Inj Prev. 1999;5(3):208-213.

13. Steffen K, Andersen TE, Krosshaug T et collab. ECSS position statement 2009: prevention of acute sports injuries. Eur J Sport Sci. 2010;10(4):223-236.

14. Harmon KG, Drezner JA, Gammons M et collab. American Medical Society for Sports Medicine position statement: concussion in sport. Clin J Sport Med. 2013;23(1):1-18. doi: 10.1097/JSM.0b013e31827f5f93

15. Jordan BD. The clinical spectrum of sportrelated traumatic brain injury. Nat Rev Neurol. 2013;9(4):222-230. doi: 10.1038/ nrneurol.2013.33
16. Abrahams S, Mc Fie S, Patricios J, Posthumus M, September AV. Risk factors for sports concussion: an evidence-based systematic review. Br J Sports Med. 2014;48(2):91-97. doi: 10.1136/bjsports-2013-092734

17. McCrory P, Meeuwisse W, Aubry M et collab. Consensus statement on concussion in sport: the 4th International Conference on Concussion in Sport held in Zurich, November 2012. Br J Sports Med. 2013; 47(5):250-258. doi: 10.1136/bjsports-2013092313

18. McIntosh AS, Andersen TE, Bahr $\mathrm{R}$ et collab. Sports helmets now and in the future. Br J Sports Med. 2011;45(16):12581265. doi: 10.1136/bjsports-2011-090509

19. Field M, Collins MW, Lovell MR, Maroon J. Does age play a role in recovery from sports-related concussion? A comparison of high school and collegiate athletes. J Pediatr. 2003;142(5):546-553.

20. Kirkwood MW, Yeates KO, Wilson PE. Pediatric sport-related concussion: a review of the clinical management of an oft-neglected population. Pediatrics. 2006;117(4):13591371.

21. Sim A, Terryberry-Spohr L, Wilson KR. Prolonged recovery of memory functioning after mild traumatic brain injury in adolescent athletes. J Neurosurg. 2008;108(3):511516. doi: $10.3171 / \mathrm{JNS} / 2008 / 108 / 3 / 0511$

22. Broshek DK, Kaushik T, Freeman JR, Erlanger D, Webbe F, Barth JT. Sex differences in outcome following sports-related concussion. J Neurosurg. 2005;102(5):856863.

23. Covassin T, Swanik CB, Sachs ML. Sex differences and the incidence of concussions among collegiate athletes. J Athl Train. 2003;38(3):238-244.

24. Dick RW. Is there a gender difference in concussion incidence and outcomes? $\mathrm{Br} \mathrm{J}$ Sports Med. 2009 May;43 Suppl 1:i46-50. 10.1136/bjsm.2009.058172 